\title{
The old globular cluster system of the dlrr galaxy NGC 1427A in the Fornax cluster ${ }^{\star}$
}

\author{
I. Y. Georgiev ${ }^{1,2}$, M. Hilker ${ }^{1}$, T. H. Puzia ${ }^{2}$, J. Chanamé $e^{3,2}$, S. Mieske ${ }^{1,4}$, \\ P. Goudfrooij ${ }^{2}$, A. Reisenegger ${ }^{5}$, and L. Infante ${ }^{5}$ \\ 1 Argelander-Institut für Astronomie ${ }^{\star \star}$, Universität Bonn, Auf dem Hügel 71, 53121 Bonn, Germany \\ e-mail: georgiev@stsci .edu \\ 2 Space Telescope Science Institute, 3700 San Martin Drive, Baltimore, Maryland 21218, USA \\ 3 Department of Astronomy, The Ohio State University, 140 West 18th Avenue, Columbus, OH 43210, USA \\ ${ }^{4}$ European Southern Observatory, Karl-Schwarzschild-Strasse 2, 85748 Garching bei München, Germany \\ 5 Departamento de Astronomía y Astrofísica, P. Universidad Católica de Chile, Casilla 306, Santiago 22, Chile
}

Received 19 January 2006 / Accepted 3 February 2006

\begin{abstract}
We present a study of the old globular cluster (GC) population of the dwarf irregular galaxy NGC 1427A using multi-wavelength VLT observations in $U, B, V, I, \mathrm{H}_{\alpha}$ and $J$ bands under excellent observing conditions. We applied color and size selection criteria to select old GC candidates and made use of archival ACS images taken with the Hubble Space Telescope to reject contaminating background sources and blended objects from the GC candidates' list. The $\mathrm{H}_{\alpha}$ observations were used to check for contamination due to compact, highly reddened young star clusters whose colors and sizes could mimic those of old GCs. After accounting for contamination we obtain a total number of $38 \pm 8 \mathrm{GC}$ candidates with colors consistent with an old $(\sim 10 \mathrm{Gyr})$ and metal-poor $\left(Z<0.4 \times Z_{\odot}\right)$ population as judged by simple stellar population models. Our contamination analysis indicates that the density distribution of GCs in the outskirts of the Fornax central cD galaxy NGC 1399 may not be spherically symmetric. We derive a present-day specific frequency $S_{\mathrm{N}}$ of 1.6 for NGC 1427A, a value significantly larger than what is observed in the Local Group dwarf irregular galaxies and comparable with the values found for the same galaxy types in the Virgo and Fornax clusters. Assuming a universal globular cluster luminosity function turnover magnitude, we derive a distance modulus to NGC 1427A of $31.01 \pm 0.21$ mag which places it $\sim 3.2 \pm 2.5$ (statistic) \pm 1.6 (systematic) Mpc in front of the Fornax central cD galaxy NGC 1399. The implications of this result for the relationship between NGC 1427A and the cluster environment are briefly discussed.
\end{abstract}

Key words. galaxies: individual: NGC 1427A - galaxies: irregular - galaxies: star clusters - galaxies: dwarf

\section{Introduction}

Old Globular Clusters (GCs) are complexes of stars which have been formed almost simultaneously out of material with basically the same initial chemical composition. They form during major star formation episodes in virtually every galaxy. Hence they are regarded as a fossil record of the initial conditions of the early host galaxy formation history.

With respect to the formation and/or assembly of early-type galaxies and their globular cluster systems (GCSs) three main competing scenarios emerged. The first assumes a hierarchical build-up of massive galaxies from a (large) population of pregalactic gaseous cloud fragments. In this scenario two major epochs of star formation occurred, with both the blue (metalpoor) and the red (metal-rich) GC populations formed in situ with a pause in between the two bursts of GC formation (Forbes et al. 1997). Another possibility, within the same scenario, is

\footnotetext{
* Based on observations obtained at the European Southern Observatory, Chile (Observing Programme 70.B-0695). Partly based on archival data of the NASA/ESA Hubble Space Telescope, which is operated by AURA, Inc., under NASA contract NAS 5-26555.

$\star \star$ Founded by merging of the Sternwarte, Radioastronomisches Institut and Institut für Astrophysik und Extraterrestrische Forschung der Universität Bonn.
}

to have a long series of smaller star-forming events with the metal-poor clusters as first generation and the metal-rich ones formed in mini-mergers at high redshifts (Beasley et al. 2002). The second scenario regards major mergers of disk galaxies (Schweizer 1987; Ashman \& Zepf 1992) in which the metalpoor GC population form early in "Searle-Zinn" fragments in the halos of the progenitor disk galaxies, while most of the metalrich GCs form during later progenitor mergers, and thus are younger. The third scenario, dissipationless satellite accretion, assumes that the host galaxy forms by classical monolithic collapse and then accretes smaller galaxies (Côté et al. 1998). The GCs of these dwarf galaxies are being captured and/or tidally stripped (Hilker et al. 1999; Côté et al. 2002). For a thorough discussion of the GCS formation scenarios we refer the reader to the reviews by Ashman \& Zepf (1998), Carney \& Harris (1998), Elmegreen (1999), Kissler-Patig (2000), van den Bergh (2000) and Harris (2003).

In all these models, the GCSs of low-mass dwarf galaxies, the most numerous galaxy type in galaxy clusters (Sandage 2005, and references therein), are envisioned as the building blocks of the GCSs of the more massive galaxies. At present the role of the GCSs of dIrr galaxies in the frame of galaxy formation is unclear. A recent study by Sharina et al. (2005) compared the GCS properties of $\mathrm{dE}, \mathrm{dSph}$, and dIrr in field and group 
Table 1. Log of observations.

\begin{tabular}{clcccc}
\hline \hline Date & Filter & $\begin{array}{c}\text { Center position } \\
\text { RA; Dec (J2000) }\end{array}$ & $\begin{array}{c}\text { Exp.time } \\
{[\mathrm{s}]}\end{array}$ & $\begin{array}{c}F W H M \\
{[\text { pixels] }}\end{array}$ & $\begin{array}{c}\text { Seeing } \\
{\left[{ }^{\prime \prime}\right]}\end{array}$ \\
\hline $2003-09-29$ & $U$ & $03: 40: 10.0-35: 36: 57.9$ & $7 \times 1595$ & 3.0 & 0.60 \\
$2002-11-06$ & $B$ & $03: 40: 10.0-35: 36: 57.9$ & $8 \times 450$ & 2.4 & 0.48 \\
$2002-11-02$ & $V$ & $03: 40: 10.0-35: 36: 57.9$ & $10 \times 150$ & 2.9 & 0.58 \\
$2002-11-02$ & $I$ & $03: 40: 10.0-35: 36: 57.9$ & $20 \times 165$ & 2.7 & 0.54 \\
$2003-01-02$ & H $\alpha$ & $03: 40: 10.0-35: 36: 57.9$ & $6 \times 1000$ & 2.7 & 0.54 \\
$2002-10-05$ & $J$ & $03: 40: 15.0-35: 37: 14.0$ & $30 \times 40$ & 2.8 & 0.42 \\
$2002-11-06$ & $J$ & $03: 40: 05.0-35: 37: 14.0$ & $24 \times 40$ & 3.3 & 0.49 \\
\hline
\end{tabular}

environments and found that all three dwarf galaxy types host a population of old GCs with very similar $(V-I)$ colors. The mean color of this population is almost identical to the $(V-I)$ colors of the blue GC population in massive early-type galaxies. One way to address the evolutionary connection between $\mathrm{dE}, \mathrm{dSph}$, dIrr, and massive galaxies is to study the specific frequency $S_{\mathrm{N}}$ (the number of GCs $\left(N_{\mathrm{GC}}\right)$ normalized by the galaxy's luminosity, Harris \& van den Bergh 1981, $\left.S_{\mathrm{N}}=N_{\mathrm{GC}} 10^{0.4\left(M_{V}+15\right)}\right)$ of such galaxies. Generally, the mean $S_{\mathrm{N}}$ values of $\mathrm{dE}, \mathrm{dSph}$, and dIrr galaxies in the Virgo and Fornax cluster are comparable (Seth et al. 2004) and very similar to $S_{\mathrm{N}}$ values of giant elliptical galaxies, which implies that all form GCs in the same proportion (per galaxy magnitude). However, the $S_{\mathrm{N}}$ of dwarf galaxies in clusters is significantly higher than for $\mathrm{dE}, \mathrm{dSph}$, and dIrr in the field environment (e.g. $S_{\mathrm{N}}=0.5$ for LMC, Harris 1991). The mechanisms that drive the transition between the GCSs of field and cluster dwarf galaxies are unclear.

Here we study the globular cluster systhem of NGC 1427A, which is the brightest dwarf irregular galaxy in the Fornax galaxy cluster. Throughout this work we adopt a distance modulus of $(m-M)=31.39 \pm 0.20 \mathrm{mag}(19 \pm 1.8 \mathrm{Mpc})$ to the Fornax cluster, as measured with Cepheids (HST Key Project, Freedman et al. 2001). NGC 1427A has a mean radial velocity of $2027.8 \pm 0.8 \mathrm{~km} \mathrm{~s}^{-1}$ (Bureau et al. 1996), which is $\sim 600 \mathrm{~km} \mathrm{~s}^{-1}$ higher than that of the cD elliptical NGC $1399\left(1430 \pm 9 \mathrm{~km} \mathrm{~s}^{-1}\right)$, located at the cluster center, and twice larger than the cluster velocity dispersion $\left(\sigma_{v}=325 \mathrm{~km} \mathrm{~s}^{-1}\right.$, Ferguson \& Sandage 1990). Such a large peculiar velocity is shared also by other cluster members, such as NGC 1404, as shown by the double-peaked redshift distribution of the Fornax cluster and the distinct group of dwarf galaxies falling toward the cluster center (Drinkwater et al. 2001). NGC 1427A shows a ring-like pattern of star formation with prominent distinct starburst complexes ionizing their immediate surroundings. One of the first studies of NGC 1427A by Hilker et al. (1997), relying on its morphological appearance and the apparent proximity to the cluster center, suggested that the interaction with the cluster environment is responsible for the observed active star formation and its future morphological evolution. Cellone \& Forte (1997) envisioned a collision with one of the many dwarf ellipticals populating the cluster center to be responsible for the ring-like appearance of NGC 1427A and speculated that the bright complex in the Northern part of the galaxy could be the intruder. A study by Chanamé et al. (2000) of the ionized gas kinematics of NGC 1427A showed that this Northern object shares the same general kinematical pattern as the rest of the galaxy body, hence making unlikely its external origin. They also argued that the most likely scenario explaning the morphological features of NGC 1427A is due to its passage through the hot intracluster medium of Fornax. Overall, this galaxy represents an ideal target to probe the influence of the cluster environment on its globular cluster population. In order to more firmly study the role of the dwarf galaxies in the context of galaxy and GCS formation scenarios, and the environment impact on their GCSs, a larger sample of dwarf galaxies should be considered, which is our goal for a future study.

In Sect. 2 we describe the observational data and its reduction. Section 3 is devoted to the globular cluster candidate selection and contamination estimates, and in Sect. 4 we analyse the properties of the selected GC candidates. Finally, in Sect. 5 the main conclusions are presented.

\section{Observations, reduction and photometry}

\subsection{Observational data}

Deep optical images of NGC 1427A in $U, B, V, I$, and $\mathrm{H} \alpha$ band passes were taken with the Focal Reducer and Low Dispersion Spectrograph 1 (FORS1) on the UT2 of the ESO Very Large Telescope (VLT) at Cerro Paranal, Chile. In addition, nearinfrared (NIR) images in $J$-band $(1.5 \mu \mathrm{m})$ were taken with the Infrared Spectrometer And Array Camera (ISAAC) attached to the UT1 of the ESO/VLT. The complete NIR data set includes $H$ and $K_{\mathrm{s}}$ imaging, however only the $J$-band was deep enough to perform this study. The FORS1 instrument has a $2048 \times 2048$ CCD detector with $0{ }^{\prime} 2 /$ pixel resolution providing a $6.8 \times 6.8$ field of view. The ISAAC imager is equipped with $1024 \times 1024$ array with pixel scale of $0.15 /$ pixel covering a $2.5 \times 2.5$ field. Two adjacent fields were taken in the NIR in order to cover NGC 1427A. All observations of NGC 1427A were performed under photometric conditions.

The observation dates, the exposure times and the average seeing are listed in Table 1 . The seeing (FWHM) was measured on the combined final images using the $\operatorname{IRAF}^{1}$ routine PSFMEASURE.

The six $\mathrm{H} \alpha$ images were obtained with the FORS1 $\mathrm{H} \alpha / 2500+60$ filter with central wavelength at $\lambda=6604 \AA$ and $F W H M=64 \AA$. Given the radial heliocentric velocity of $V_{r}=2027.8 \pm 0.8 \mathrm{~km} \mathrm{~s}^{-1}$ (Bureau et al. 1996), NGC 1427A is placed at redshift $z=0.00676$. Hence, the $\mathrm{H} \alpha$ emission lines of NGC 1427A (rest frame wavelength at $6563 \AA$ ) are redshifted by $44 \AA$, which is $3 \AA$ above the central wavelength of the $\mathrm{H} \alpha$ filter used.

\subsection{Reduction and photometry}

The basic image reduction was performed in a standard way using IRAF software packages. The bias subtraction and flat

1 IRAF is distributed by the National Optical Astronomy Observatories, which are operated by the Association of Universities for Research in Astronomy, Inc., under cooperative agreement with the National Science Foundation. 


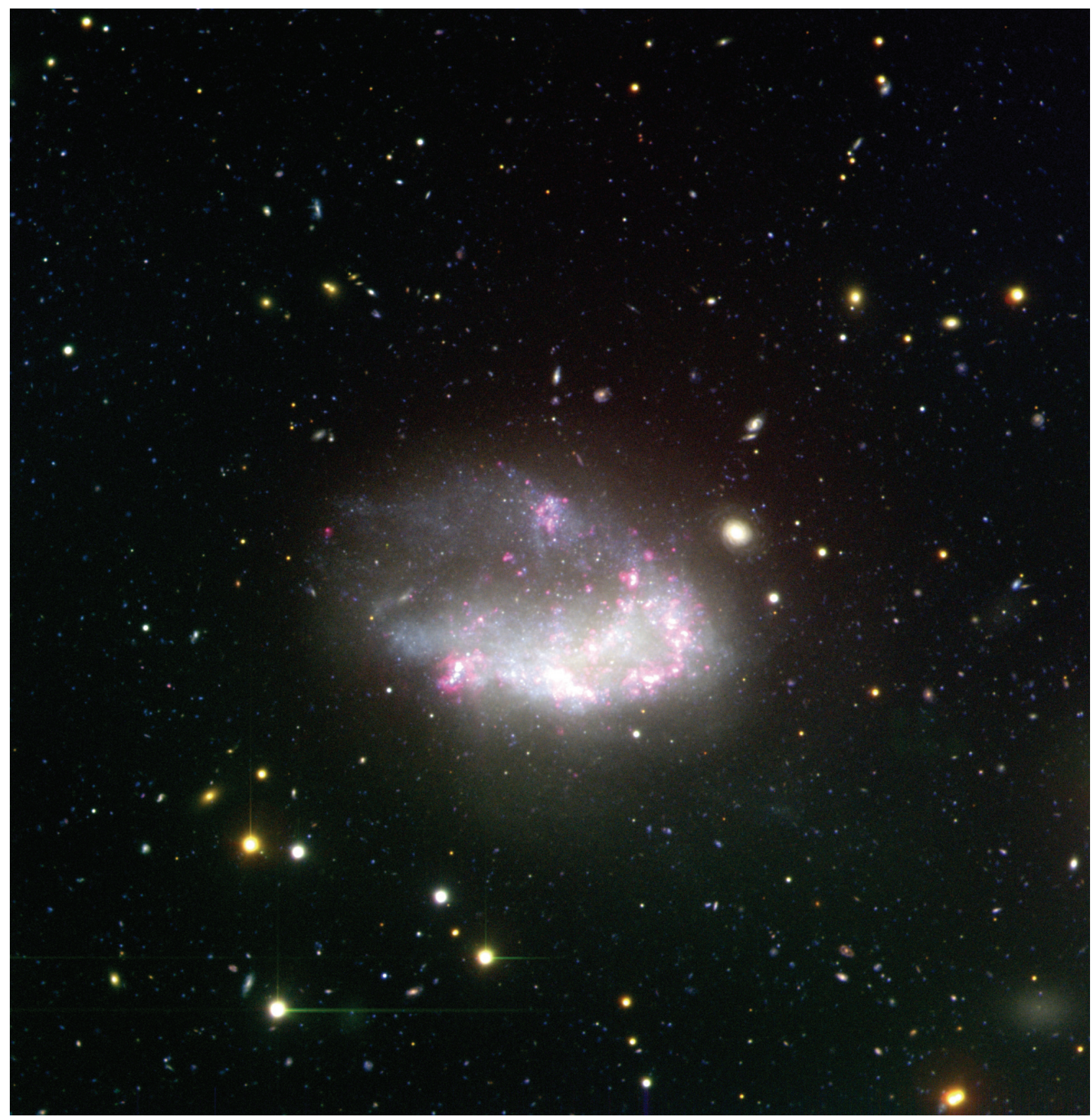

Fig. 1. NGC 1427A color composite image. For the blue, green and red channels the $U, V$ and $\mathrm{H} \alpha$ filters were used, respectively. North is up, East is on the left. The entire field of view of this image is $\sim 6$ ' $7 \times 6$ '.7 which corresponds to $\sim 37 \times 37 \mathrm{kpc}$ at the Fornax Cluster distance.

field corrections were applied using the CCDRED package taking into account the CCD noise parameters. The IR images were taken in the usual object-sky-object-sky sequences. Both object and sky exposures were bias- and flatfield-corrected in the same manner. The sky images were cleaned from all detected sources with a SExtractor object map, and object residuals were manually replaced with nearby empty sky patches. Then, these cleaned sky images were subtracted from the associated object exposures to obtain a background corrected science frame. Before combination, each single exposure in each filter was shifted by integer pixels to a defined reference image to provide the image flux conservation during the transformations. For cosmic rays rejection we used the laplacian cosmic-ray identification algorithm described and provided as IRAF cl-script ${ }^{2}$

${ }^{2}$ http://www.astro.yale.edu/dokkum/lacosmic by van Dokkum (2001). Examination of the output rejected pixel map showed that this routine did a nice work cleaning all cosmic rays without touching the sharp tips of the point sources. Given the sharpness of the images (see FWHM in Table 1) this was a major problem when using the IRAF built-in rejection algorithms, which cut the stars' tips, during the image combining process. This is why we preferred first to clean the reduced images in each filter and then to combine them to a single image without rejection algorithms. From the final average combined images a color image of NGC 1427A was produced (Fig. 1) using $U, V$ and $\mathrm{H} \alpha$ filters.

Point sources in NGC 1427A were identified using the DAOFIND routine of the DAOPHOT package (Stetson 1987), adopting a detection threshold of $5 \sigma$ above the sky level. Lower thresholds were found to give too many spurious detections. 


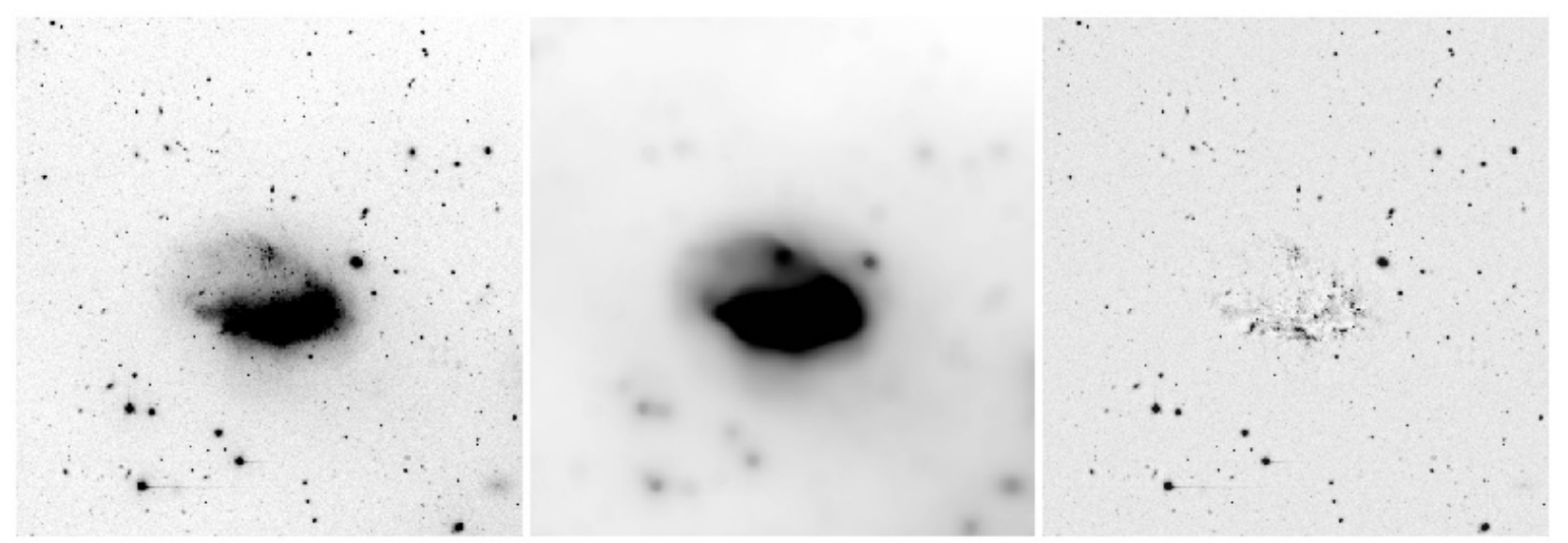

Fig. 2. Left: $V$ band image of NGC 1427A. Middle: median-filter smoothed image (using smoothing kernel of 41 pixels radius). Right: median-filter subtracted residual image.

Table 2. Listed are the PSF magnitude corrections $\left(r_{\text {ap }}=16 \rightarrow \infty\right)$; the accuracy of the fits to the transformation equations and the completeness limits in $U, B, V, I$ and $J$ bands.

\begin{tabular}{ccccccc}
\hline \hline Filter & $U$ & $B$ & $V$ & $I$ & \multicolumn{2}{c}{$J$} \\
& & & & & Field1 & Field2 \\
\hline Corr. & -0.099 & -0.058 & -0.087 & -0.074 & -0.074 & -0.057 \\
rms & 0.058 & 0.042 & 0.023 & 0.026 & 0.050 & 0.045 \\
$90 \%$ & 26.21 & 26.95 & 25.65 & 25.66 & 21.12 & 20.67 \\
$50 \%$ & 26.85 & 27.41 & 26.55 & 26.28 & 21.99 & 21.59 \\
\hline
\end{tabular}

Visual image inspection showed that no objects were missed and only few false detections were included in the lists.

In order to improve the faint object photometry the underlying and extended galaxy light was subtracted form the original images. This was done with a ring aperture median filtering on images from which bright objects had been previously subtracted. A smoothing kernel of 41 pixels radius proved to give the best results, i.e., the wings of the bright, well-exposed stars were not affected. This was confirmed by the comparison between magnitudes of stars measured in the subtracted and nonsubtracted images (see below). The filtered images in each passband were subtracted from the original ones, and the resulting images then were used for photometry. Due to the complex structure of this irregular galaxy it was impossible to fit and subtract a simple model.

An example of the $V$-band images passed through the filtering procedure is shown in Fig. 2. With this choice of ring radius only the brightest overexposed foreground stars and extended background galaxies were affected. The residual image on the right panel was used for the final photometry. In order to obtain reliable photometry for all objects, especially those in the most crowded regions, a point-spread function (PSF) photometry was performed. To create the PSF model we selected between 7 and 15 (depending on the filter) well-isolated PSF stars. The PSF photometry was performed with the DAOPHOT/ALLSTAR procedure.

To account for the star light lost due to the PSF model fitting and smoothed image subtraction, the differences between the aperture photometry and the PSF photometry magnitudes were determined using curves of growth for various aperture sizes in all filters. These curves of growth proved that the median filtering, with this choice of of kernel raduis, did not affect the stellar profiles significantly. Hence, the correction to be applied is mainly due to PSF-model fitting effects. We adopted a radius of 16 pixels (corresponding to $>5 \times F W H M$ ) for all filters. The corrections are listed in Table 2.

Photometric calibrations of each data set from instrumental to standard magnitudes were performed in a standard way using Landolt (1992) standard stars which were observed in the corresponding nights. After fitting the zero points, extinction coefficients and color terms (as given by the ESO Quality Control for the corresponding nights), the resultant rms of the fits for the $U B V I$ and $J$ photometry are shown in Table 2 .

The main objects of interest for the current paper are the old globular clusters associated with NGC 1427A. At the adopted distance of the Fornax Cluster, one FORS1 pixel (0.'2) corresponds to $\sim 19 \mathrm{pc}$ projected size. Given the mean $F W H M$ ( $\leq 3$ pixels) of our images all compact sources associated with NGC 1427A (SCs, rich OB associations, compact H II regions, or very luminous giant stars) are expected to have a star-like profile and the adopted PSF photometry is a good choice.

\subsection{Completeness determination}

At the distance of the Fornax Cluster the expected universal turnover magnitude of the globular cluster luminosity function (absolute value $M_{\mathrm{V}, \mathrm{TO}} \simeq-7.4 \mathrm{mag}$; Harris 1996, 2001) is expected at $V_{\mathrm{TO}} \sim 24 \mathrm{mag}$. To estimate up to which limiting magnitude our observations are complete we used the final combined and background subtracted images and the IRAF/DAOPHOT/ADDSTAR procedure.

To avoid artificial crowding in the images we added 100 stars per image with magnitudes ranging from 21 to 29 mag in the optical and 14 to $25 \mathrm{mag}$ in the IR images in 100 runs. Thus, the total number of stars that were added per filter is 10000 . The artificial objects were generated from the PSF of each correspondent field. Detection and photometry of the artificial objects was performed in the same manner as described in Sect. 2.2. The recovered number of objects divided by the number of the input artificial stars then gave us the completeness fraction. In Fig. 3 we show the completeness functions for the optical and IR data. The completeness is a function of the background level, hence it is expected to be variable toward the central galaxy regions. This effect should be accounted for if the photometric study is devoted mainly to the objects in those inner most regions. However, the GCs which we study here are typically located at large galactocentric distances and this effect is expected to be not so significant. The long exposure times and the photometric conditions resulted in deep images in the optical 


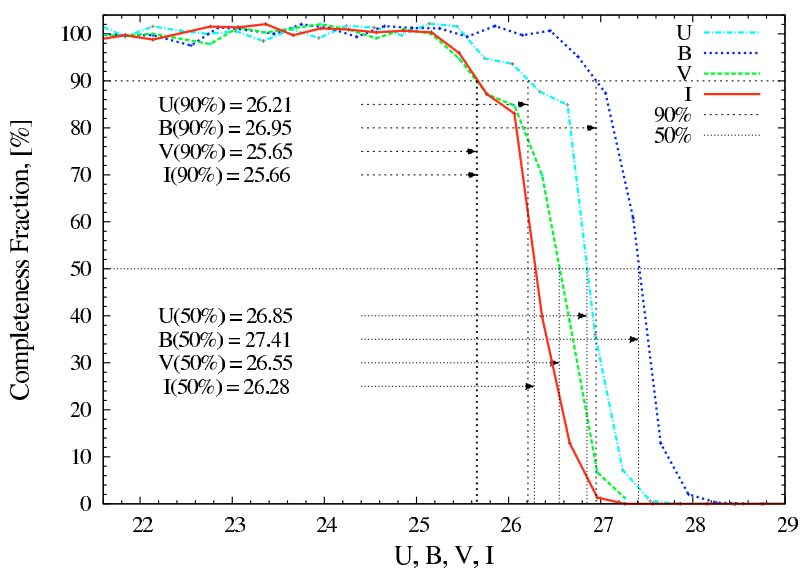

Fig. 3. Completeness functions estimated with the add-star experiment.

wavelengths (see Fig. 3 and Table 2). In the $U B V$ and $I$-bands we easily reach $>1$ mag beyond the expected turnover of the GC luminosity function, while in the near-IR we reach only its bright end (e.g., $M_{\mathrm{J}, \mathrm{TO}}=-9.21 \mathrm{mag}$; Barmby et al. 2001, thus $J_{\mathrm{TO}} \simeq 22.2 \mathrm{mag}$ ).

\section{4. $H_{\alpha}$ calibration and results}

The calibration of the $\mathrm{H} \alpha$ images was based on the spectrophotometric standard star LTT 1020 (Hamuy et al. 1992, 1994). As initial guess for the extinction coefficient we used the observatory value for the $R$ filter and then computed our zero point. Using the magnitude and luminosity of LTT1020 we obtained the following relation

$m_{\mathrm{H}_{\alpha}}=-2.5 \times \log \left(F_{\mathrm{H} \alpha}\right)-21.51 \pm 0.11$

where $F_{\mathrm{H} \alpha}$ is the flux in $\operatorname{erg~s}^{-1} \mathrm{~cm}^{-2} \AA^{-1}$. This relation was subsequently used to convert from magnitude to $\mathrm{H}_{\alpha}$ luminosity in order to derive the star formation rate (SFR) for NGC 1427A (see below).

The raw $\mathrm{H} \alpha$ images were reduced, aligned, registered, and a final combined and background subtracted $\mathrm{H} \alpha$ image was produced (Sect. 2.2). In order to subtract the continuum emission from our $\mathrm{H} \alpha$ image we follow Knapen et al. (2004) and use as reference the $I$-band image (due to the lack of a $R$-band image). To determine the scaling factor, which tells how the continuum image must be scaled to match the intensity level of the continuum emission in the $\mathrm{H} \alpha$ image, we followed the approach described by Böker et al. (1999).

We applied the so determined scaling relation to the $I$-band image and subtracted it from the $\mathrm{H} \alpha$ image. This gave us the resulting pure $\mathrm{H} \alpha$ emission-line image. As expected, sources without $\mathrm{H} \alpha$ emission disappeared to the background level whereas sources having $\mathrm{H} \alpha$ in absorption were over-subtracted. A check for young embedded star clusters or compact H II regions contaminating the GC candidate sample revealed no emission in excess or in absence at the GC candidate's positions on the residual $\mathrm{H}_{\alpha}$ images. Our conclusion is that the selected GC candidates are hardly if at all polluted by young compact sources from the inner starburst galaxy regions.

The brightest background/foreground objects were masked out on the so obtained residual $\mathrm{H} \alpha$ images and the $\mathrm{H} \alpha$ magnitude of the galaxy was measured using the IRAF/STSDAS task ELLIPSE. For the conversion from magnitude to luminosity we used the adopted distance of $19 \mathrm{Mpc}$ (Freedman et al. 2001). The star formation rate was derived from the $\mathrm{H} \alpha$ flux using the improved relation given by Hunter \& Elmegreen (2004):

$$
\dot{M}=5.96 \times 10^{-42} L_{\mathrm{H}_{\alpha}} 10^{0.4 A_{\mathrm{H}_{\alpha}}} M_{\odot} / \mathrm{yr}
$$

where $L_{\mathrm{H}_{\alpha}}$ is the $\mathrm{H}_{\alpha}$ flux in erg/s, $A_{\mathrm{H}_{\alpha}}=0.811 A_{\mathrm{V}}$ is the extinction coefficient in $\mathrm{H}_{\alpha}$. We derived $\dot{M}=0.057 M_{\odot} /$ yr. This is a typical value for a starburst dIrr galaxy (Hunter \& Elmegreen 2004; Seth et al. 2004). This estimate should be regarded as an upper limit for the SFR since, due to the lack of flux calibrated emission-line spectra for N 1427A, no care was taken for the N II 6548,6583 emission-line doublet, which partly falls within the $\mathrm{H}_{\alpha}$ filter pass-band.

\subsection{Foreground and intrinsic extinction}

Absorption caused by the Galactic interstellar medium (ISM) that affects the objects' magnitudes, hence colors, have to be taken into account in order to compare the object's colors (magnitudes) with those of stellar evolutionary models (Sect. 3.1).

In order to calculate the extinction correction towards NGC 1427A for each filter we adopted ${ }^{3} E(B-V)=0.012$ (Schlegel et al. 1998), $A_{\mathrm{V}}=3.1 \times E(B-V)$, formulae (2) and (3) in Cardelli et al. (1989) and the central wavelengths for the FORS1 and ISAAC filters taken from the ESO web page.

Although the purpose of the $J, H, K$ imaging was to determine the extinction map of NGC 1427A, the images turned out to be too shallow for this goal. Therefore, we can not apply individual internal reddening correction, which could be large for clusters located inside or behind the galaxy body. Nevertheless, we do not expect this to be a significant effect because of the following reasons. First, as will be seen in Fig. 9, we observe $\sim 10-15$ candidates in the innermost galaxy regions, and only a fraction of these are expected to be inside or behind, and therefore affected by internal extinction. Second, James et al. (2005), studying the extinction on the $\mathrm{H}_{\alpha}$ flux as a function of the galaxy type, show that the mean extinction correction for dIrrs is $A_{\mathrm{H}_{\alpha}} \simeq 0.4 \mathrm{mag}$, which transforms to $E_{B-V} \simeq 0.06$ (or $V-I \simeq 0.07 \mathrm{mag}$ ). The overall internal reddening therefore is expected to be small and this is why we consider in the following only the foreground extinction.

\section{Globular cluster candidates selection}

Our final photometry list contains stars, star clusters and extended objects (galaxies, H II regions, OB-associations, etc.). In order to select globular cluster candidates we apply color, magnitude and size selection criteria according to the expected properties of these old stellar systems at the distance of NGC 1427A.

\subsection{Color and magnitude selection}

The globular cluster candidate selection is mainly based on the colors of the objects in the color-color diagrams. The adopted distance modulus to the Fornax cluster limits the expected brightest globular cluster magnitude to be $m_{V} \simeq 21$ mag assuming $M_{V}=-10.29$ for the brightest Milky Way (MW) globular cluster $\omega$ Centauri (Harris 1996).

To constrain a region of typical GC colors we use the 2003 updated version of the Harris (1996) catalog of MW GCs ${ }^{4}$. The color selection limits, which we chose as first selection criterion, are defined as an ellipse that approximately encompasses the colors within $-0.16 \leq(U-B) \leq 0.7$ and $0.72 \leq(V-I) \leq 1.4$ (see

\footnotetext{
3 http://nedwww.ipac. caltech.edu/

4 http://www . physics.mcmaster.ca/Globular.html
} 

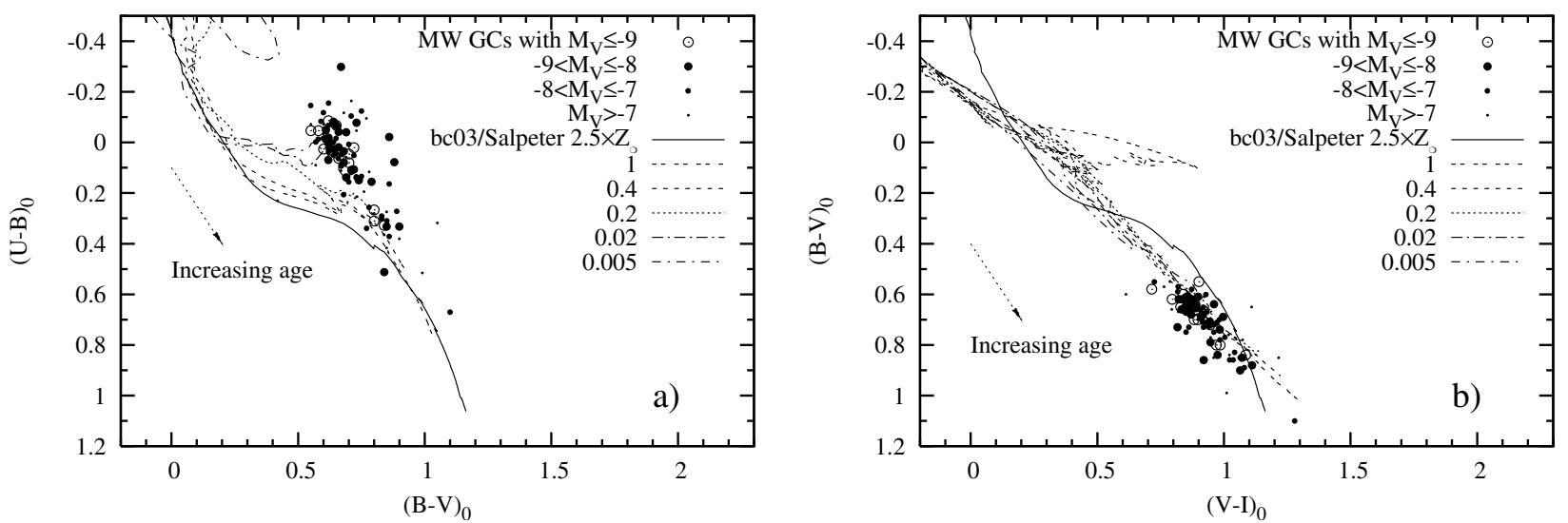

Fig. 4. Milky Way globular clusters' $(U-B)_{0}$ vs. $(B-V)_{0}$ a) and $(B-V)_{0}$ vs. $(V-I)_{0}$ b) color-color "showing the power of a color-color selection". (readers can see that anyway) diagrams. The clear metallicity separation of the MW globular cluster system can easily be seen in a), while in b) the objects' metallicity separation smears out and the SSP models for different metallicities are indistinguishable.

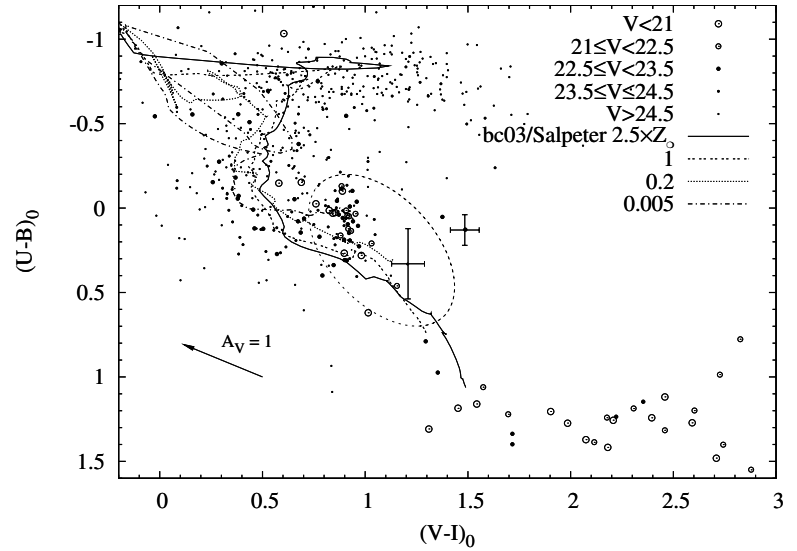

Fig. 5. $(U-B)_{0}$ vs. $(V-I)_{0}$ diagram showing the colors of all point sources (Sect. 3.2) found in the FORS1 field. The different point types indicate objects with different magnitudes. The arrow shows the magnitude and the direction that would have to be applied to the colors of objects subject to an intrinsic extinction of $A_{\mathrm{V}}=1 \mathrm{mag}$. With lines are plotted the Bruzual \& Charlot (2003) SSP models for different metallicities. Representative photometric errors are shown for two GCs with luminosities around the expected GCLF turnover magnitude.

Fig. 5). These color ranges are found to be typical for GCs in a large variety of galaxy types (e.g. Ashman \& Zepf 1998; Larsen et al. 2001; Lotz et al. 2004; Chandar et al. 2004; Sharina et al. 2005, and ref's therein).

The $(U-B)$ vs. $(V-I)$ and the $(U-B)$ vs. $(B-V)$ color combinations proved to be the most distinctive when disentangling ages and metallicities. Any color combination not including the $U$-band makes the models almost indistinguishable (compare the two panels in Fig. 4), hence leading to large uncertainties in assessing ages and/or metallicities. Due to line blanketing effects in the stellar atmospheres the $(U-B)$ color is very sensitive to metallicity variations. Therefore, inclusion of the $U$-band in photometric studies aiming at accurate age/metallicity determinations is very important. A detailed discussion on the right filter selection when comparing observations with evolutionary synthesis models is provided by Anders et al. (2004). Indeed, as shown in Fig. 4, the well established bimodal metallicity distribution of the MW globular cluster system (e.g., van den Bergh 1993; Carney \& Harris 1998; Mackey \& van den Bergh 2005, and references therein) can easily be seen when using $(U-B)$ color.
In Fig. 5 we show the colors of all point sources detected in the FORS1 field, corrected only for foreground Galactic extinction. The different symbol types indicate different $V$-band magnitudes of the objects. The arrow shows the direction and the magnitude with which the color of an object would have to be shifted if an extinction of $A_{\mathrm{V}}=1 \mathrm{mag}$ intrinsic to NGC 1427A is applied.

Representative photometric errors are shown in Fig. 5 to demonstrate the accuracy of our photometry for objects in the magnitude bins around the expected GC luminosity function turnover at $\sim 24$ mag and obeying the GC candidate selection criteria (see Sect. 3.2).

In the color-color diagram one can see a large number of blue objects. Examination of the positions of these objects showed that most of them are found in the inner starburst regions of NGC 1427A. The rest of the blue objects are associated with faint sources, likely background galaxies and sources associated with them: an example is the background spiral galaxy westward of NGC 1427A (Fig. 1). The visual inspection of the observed very red and bright objects with $(V-I)>1.5$ showed that they are saturated foreground stars and likely background elliptical galaxies. In the color region where GCs are expected a high density of objects is observed.

\subsection{Point source selection criterion}

As pointed out in Sect. 2.2 the distance to NGC 1427A puts an additional constraint to the expected sizes of the GCs associated with NGC 1427A. The typical GC half-light radii are in the range 1 to 20 pc (e.g. Kundu \& Whitmore 2001; van den Bergh \& Mackey 2004; Jordán et al. 2005). Given the adopted distance to the Fornax cluster, 1 pixel of our images corresponds to $\sim 19 \mathrm{pc}$ projected size. Therefore, GCs are expected to be unresolved and to have point source radial profiles. As point source selection criterion we used the IRAF ALLSTAR task output parameter "sharp". Sharp is computed during the fit of the PSF model and estimates the intrinsic angular size of the object. It is roughly defined as the difference between the square of the width of the object and the square of the width of the PSF. It has values close to zero for unresolved point sources, large positive values for blended sources and partially resolved galaxies and large negative values for cosmic rays and blemishes.

As first selection criterion we used the color-color limits explained in Sect. 3.1. Then for the color-color selected objects we plotted the $V$-band sharp value against $V_{0},(U-B)_{0}$ and $(V-I)_{0}$. 


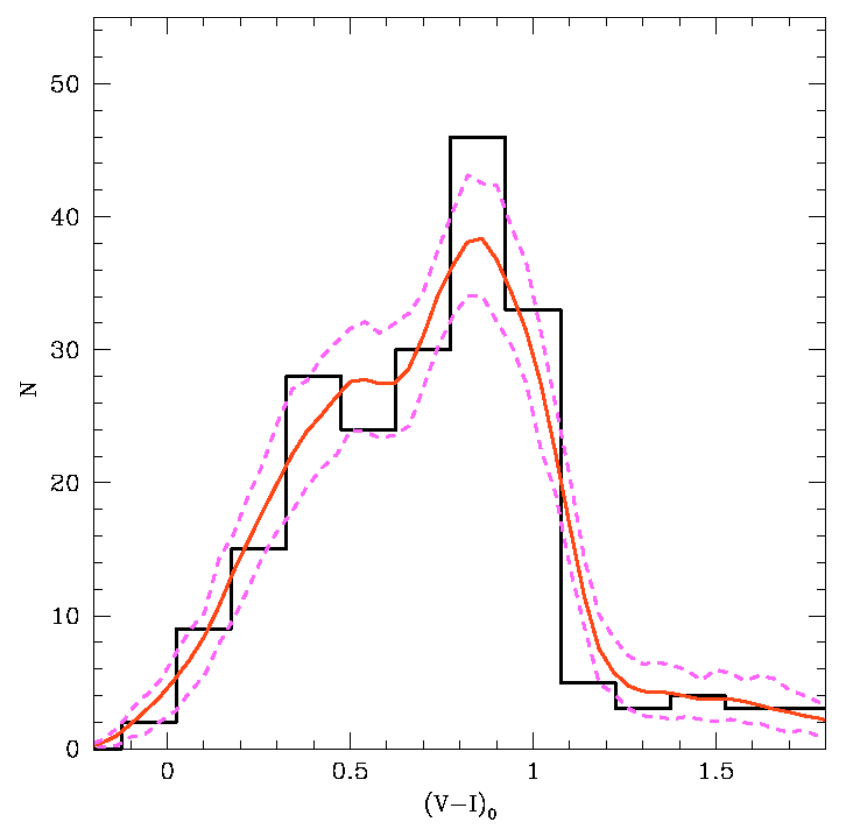

Fig. 6. $(V-I)_{0}$ color distribution of all point sources with $(U-B)_{0} \geq$ -0.3 (see Fig. 5). The $U-B$ color cutoff was introduced to lower the contamination from faint or objects with highly uncertain photometry in the starburst regions. The Epanechnikov-kernel probability density estimate and its $90 \%$ confidence limits are plotted with thick/red and dashed/magenta lines, respectively. Similar color distributions are observed in star cluster systems of field dIrrs Sharina et al. (2005).

Plots of the sharp values for the other filters were produced and showed insignificant differences. However, such differences might be expected between $U$-band and $V$-band sharp values for example if in the selected color-color region highly reddened bright compact H II regions embedded in the NGC 1427A starburst regions are observed. Such objects can have different profiles in the $U$ and in the $V$ filter due to the enhanced contribution of the ionized gas in $U$-band which is invisible in $V$.

The shape of the functions defining the upper and lower cutoff sharp values were determined from the sharp value distribution of artificially added stars previously used for the completeness tests (Sect. 2.3). The scatter of the sharp value increases towards fainter magnitudes due to increasing uncertainties in the PSF fitting procedure. At approximately $V_{0}=24.3$ and $(V-I)_{0}=0.86 \mathrm{mag}$ couple of objects slightly above the sharp cut-off function are observed. A visual inspection of these objects revealed that they are somewhat extended sources embedded in the central starburst regions, which suggests at their young nature. An additional check was made using available HST/ACS images which ultimately revealed their morphology as diffuse and blended sources. The final list of our GC candidates contains 60 objects.

The $(V-I)$ color distribution for all point sources (presumably star clusters) with $(U-B) \geq-0.3$ is shown in Fig. 6 . The $U-B$ cutoff was introduced to lower the contamination from very faint unresolved amorphous objects with $V-I$ colors within the old GCCs range (compare with Fig. 5). An Epanechnikovkernel probability density estimate shows a clear indication for (at least) two characteristic $(V-I)$ color peaks at $\sim 0.4$ and 0.9 mag. These color peaks are similar to those found in the colors distributions of star cluster systems in field dIrr galaxies (Sharina et al. 2005). However, the contamination effects in our sample prevent us from a more detailed analysis.

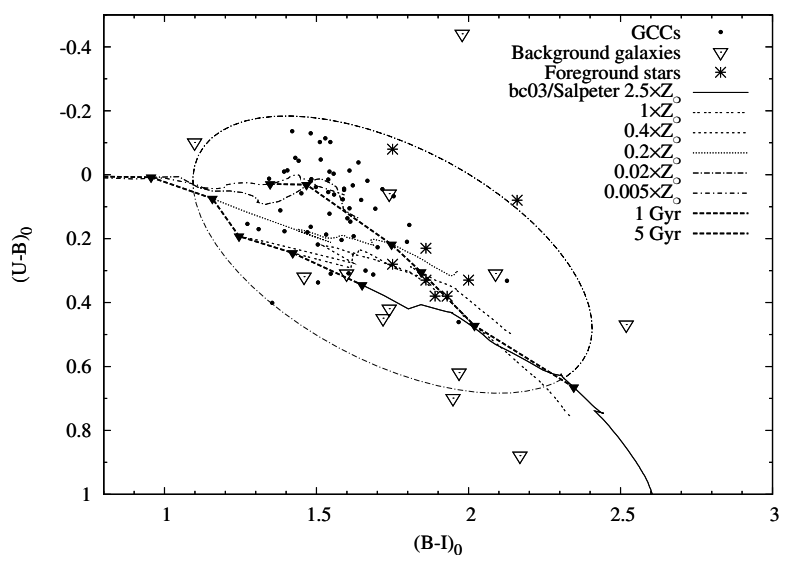

Fig. 7. $(U-B)_{0}$ vs. $(B-I)_{0}$ color-color diagram of the selected globular cluster candidates (dots), foreground stars (asterisk) and background galaxies from the FDF catalog (open triangles). The ellipse indicates the transformed GC candidate color-color selection region as indicated in Fig. 5. Lines show iso-metallicity tracks and isochrones for various ages and metallicities.

\subsection{Contamination and GC numbers}

Despite the applied globular cluster selection criteria, there can still be sources contaminating our final GC candidate sample in the selected color limits. Possibilities are faint ( $V \geq 21 \mathrm{mag}$ ) Galactic halo stars, distant and compact bulges of unresolved background galaxies, and unresolved low-redshift $(z \sim 0.1-1.0)$ starburst galaxies with ages $\leq 300 \mathrm{Myr}$ (Puzia et al. 2004). Highly reddened ( $A_{\mathrm{V}} \geq 1 \mathrm{mag}$ ) young compact sources (H II regions, OB associations, young star clusters) in the inner regions of NGC 1427A are potential contaminants as well. This is estimated in Sect. 3.3.1 below.

Another source of contamination to the list of GC candidates of NGC 1427A is contamination from possible Fornax intracluster globulars (Bassino et al. 2003) and the GC population of NGC 1399 which is thought to extend to large distances (Dirsch et al. 2003). We discuss this in Sect. 3.3.2.

\subsubsection{Foreground/background}

To estimate the foreground star contamination we used a synthetic Galaxy stellar population model $^{5}$ (Robin et al. 2003). For the specified color and magnitude ranges (Sects. 3.1 and 3.2) and within the FORS1 field of view the model predicts a total number of 7 foreground stars towards NGC 1427A (see Fig. 7).

We estimate the contamination by background galaxies using the FORS Deep Field (FDF) data (Heidt et al. 2003). The main criterion for selecting objects from the FDF cataloged as galaxies is the limiting bright magnitude cutoff $I \geq 20.1$ mag and the completeness limit of our observations in the $I$-band (Fig. 3). However, the inspection of our final globular cluster candidate sample shows that there are no candidates with $I \geq 24.5$ mag, which actually represents the faint end of the GC luminosity function. Therefore, in Fig. 7, due to the lack of $V$-band photometry in the FDF, we present the $(U-B)_{0}$ vs. $(B-I)_{0}$ color distribution of the background galaxies in the range $20.1 \leq I \leq 24.5 \mathrm{mag}$ (triangles). It can be seen that $\sim 7$ background galaxies are expected in the GC candidate color-color region.

As an attempt to identify these background galaxies, as well as contamination from blended sources in the crowded

\footnotetext{
${ }^{5}$ http://bison.obs-besancon. fr/modele/
} 


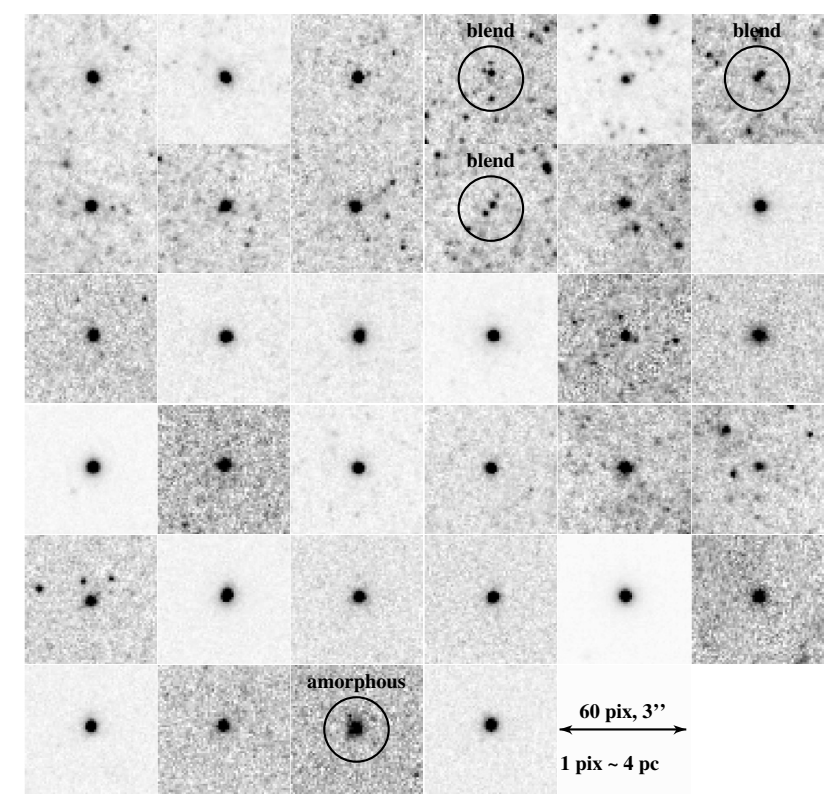

Fig. 8. F625W-band mosaic of the GC candidates as seen using HST/ACS. Circles indicate the three blends and the amorphous objects which were rejected from the original ground-based GC candidate list based on these ACS data.

galaxy regions, we take advantage of the high spatial resolution of archival HST/ACS images of NGC 1427A taken with the F625W filter (Program GO-9689, PI: M. Gregg), which were combined using MultiDrizzle (Koekemoer et al. 2002). A visual examination shows that none of the 34 GC candidates (out of 60) in common for the FORS1 and ACS fields are resolved as obvious background galaxies. The appearance of the GC candidates on the ACS chips are presented in Fig. 8. This shows that our point-source selection criteria performed well and only a fraction of the seven BG galaxies estimated above could remain unresolved by ACS. However, three of our GCCs turned out to be blended sources and one seems amorphous (possibly a compact OB-association, see Fig. 8). The latter is located in the inner starburst regions of the galaxy and passed the point-source selection criteria at the faint magnitude end. The blends and the amorphous object were excluded from subsequent analysis.

Due to the smaller field of view of HST/ACS relative to the FORS1 field, not all candidates could be examined this way, especially those far from the galaxy body. Thus we can not extrapolate the above conclusions to the rest of the candidates, and unresolved background objects could still be present among the remaining 26 GCCs. Discrimination between GCs and foreground stars is also not an easy task since one ACS pixel corresponds to $\sim 4 \mathrm{pc}$ projected size at the Fornax distance. Thus, GCs more compact than this would have a stellar appearance. Only spectroscopy can fully address the true foreground/background contamination fraction. A detailed analysis of the NGC 1427A HST/ACS data will be presented in a forthcoming study.

\subsubsection{Fornax cluster GC contamination}

Figure 9 shows the spatial distribution of GC candidates around NGC 1427A across the $V$-band FORS1 field of view. It can be seen that the number of GC candidates increases towards the galaxy's center, which suggests that they are really associated with NGC 1427A. The surface density of all GCCs is shown in Fig. 10 with red/solid histogram together with the Poisson errors.

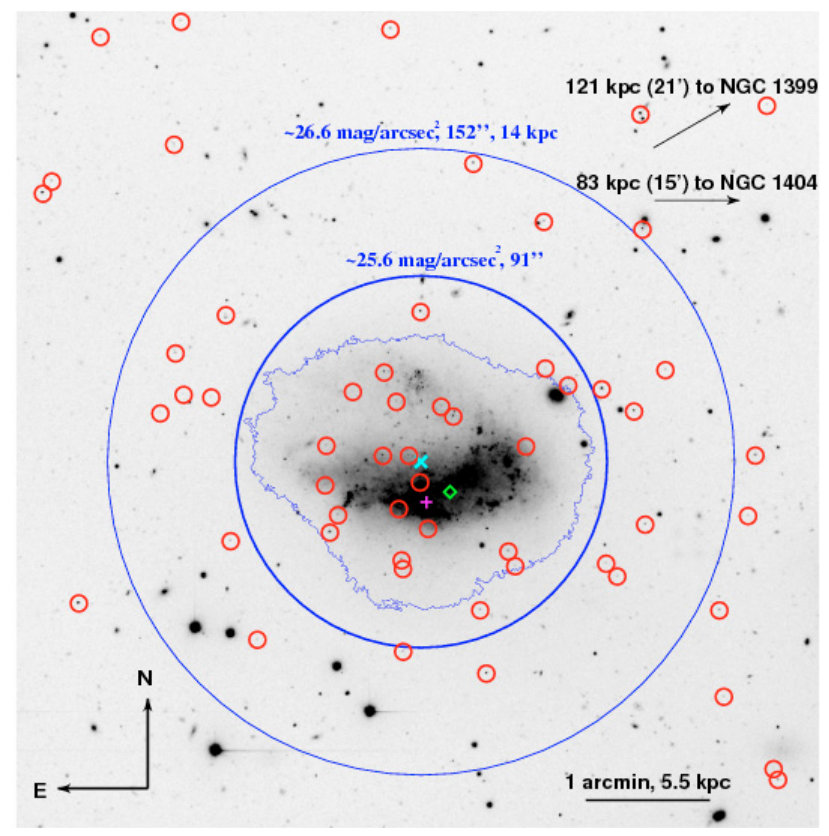

Fig. 9. 6!8 $\times 6$ 6 $^{\prime} 8 \mathrm{~V}$-band VLT/FORS1 image. The positions of the selected (see Sects. 3.2 and 3.3.1) GC candidates are indicated with (red) circles. With arrows the directions and projected distances towards the Fornax cD galaxy NGC 1399 and the elliptical NGC 1404 are shown. With a " $\times$ " the adopted center of NGC 1427A is marked, with a "+" the fitted center derived from the median smoothed image and with a diamond the kinematical center determined from the ionized gas kinematics (Chanamé et al. 2000). The two large circles approximately encompass the $\mu_{V}$ isophotes at $\mu_{V}=25.6 \mathrm{mag} / \operatorname{arcsec}^{2}$ (the true contours of that isophote is shown as well) and at $\mu_{V}=26.6 \mathrm{mag} / \operatorname{arcsec}^{2}$ (see Sect. 4.3 and Fig. 14).

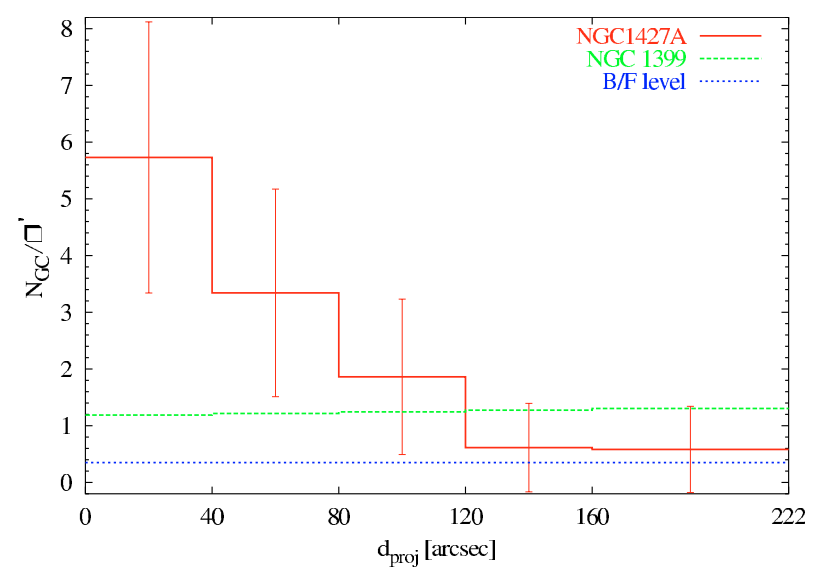

Fig. 10. Plot of the radial number density distribution of the NGC 1427A GC candidates (thick line histogram) and the correspondent Poisson errors, the expected level of contamination by the NGC 1399 GCS at the distance of NGC 1427A (dashed/green line) and the background/foreground contamination level (dotted/blue line).

However, the relatively close projected distances (see Fig. 9) between NGC 1427A and the giant elliptical galaxy NGC 1399 (22'9) and the other nearby elliptical NGC 1404 (15:7) raise the question whether all found GC candidates really belong to NGC 1427A. A study of the NGC 1399 GCS by Dirsch et al. (2003) concludes that even in their (most distant) studied field (which extends up to $\leq 23$ arcmin from NGC 1399) the number counts have not yet reached the background value. However, as they point out, at such large distances their fields could contain 
possible intracluster GCs (ICGC) as proposed by Bassino et al. (2003) in their search for GCs around dwarf elliptical galaxies in the Fornax cluster. The latter study derived an intracluster GC surface density of $\sim 0.25$ and $0.13 \mathrm{GCs} / \mathrm{arcmin}^{-2}$ at distances of $40^{\prime}$ and $110^{\prime}$ from the cluster center, respectively. The existence of intracluster globulars is further supported by recent simulations by Yahagi \& Bekki (2005).

The estimated GC number density contamination from the NGC 1399 GCS is shown as a green/dashed histogram in Fig. 10. At the distance of NGC 1427A, 22'.9, 1.26 globular clusters per $\operatorname{arcmin}^{2}$ are predicted (Dirsch et al. 2003). Figure 10 shows that at large galactocentric distance from NGC 1427A, our number counts are considerably below the expected contamination from NGC 1399, though still within the errorbars.

There are three possibilities that could account for this discrepancy between the observed and expected contamination level from NGC 1399. The first one is that we are missing a number of GCs. However, according to the completeness analysis (Sect. 2.3) we are confident that this is not the case. The second possibility is that the Dirsch et al. (2003) values might not be aplicable toward NGC 1427A, which is likely, because the fields studied by Dirsch et al. (2003) extend towards the nearby elliptical galaxy NGC 1387 (their Fig. 1) which could give rise to an enhanced GC number density. The third possibility is to suggest a non-spherical distribution of GCs around NGC 1399 or ICGCs, resulting in a lower number density towards NGC 1427A as compared to other directions. We think that a combination of the last two is the more plausible explanation. A possible GC contamination from the even closer (15:7) elliptical NGC 1404 seems to be ruled out as well, since its GCS is not as extended as that of NGC 1399. Indeed, at a distance from NGC 1404 of only 3 arcmin the number density is already $0.63 \mathrm{arcmin}^{-2}$ (Richtler et al. 1992; Forbes et al. 1998) which, when extrapolated to the position of NGC 1427A, becomes irrelevant.

The estimated total number density of background/ foreground objects (Sect. 3.3.1) shown in Fig. 10 (0.35 \pm 0.59 objects/arcmin ${ }^{2}$ ) is below the levels we reach in our outermost radial bins $\left(0.61 \pm 0.78 \mathrm{GCCs} / \operatorname{arcmin}^{2}\right)$. Although this values are within the errorbars the difference could be interpreted as: $(i)$ either the local $\mathrm{BG} / \mathrm{FG}$ is enhanced, or (ii) that there exist genuine GCs associated with either NGC 1427A, NGC 1399 or the ICGSs population at those galactocentric distances. Although the latter possibility seems more plausible to us, this is irrelevant for our purposes because the maximum contamination that we could possibly assume would be what we measure at the outermost bins (ideally containing no GCs from NGC 1427A). Thus we adopt $0.61 \pm 0.78$ counts/arcmin ${ }^{2}$ as our best-estimate for the contamination level.

\subsubsection{Final GCC number}

Recalling the total of 60 candidates (Sect. 3.2) here we determine what fraction of these are contaminating objects. As was shown in Sect. 3.3.1 we expect 7 foreground stars, which are indistinguishable from actual GCs with our data. The expected number of background galaxy contamination (Sect. 3.3.2) is a fraction of the 7 objects predicted by FDF. Therefore we end up with a number between 10 and 14 BG/FG contaminants.

In Sect. 3.3.3 we estimated a maximum density of 0.61 objects/arcmin ${ }^{2}$ contamination, which when multiplied by the $43 \operatorname{arcmin}^{2}$ FORS 1 field of view, results in 26 contaminating objects. Therefore we expect a total number of contaminants between 10 and 26. Taking the mean value of 18 and the four

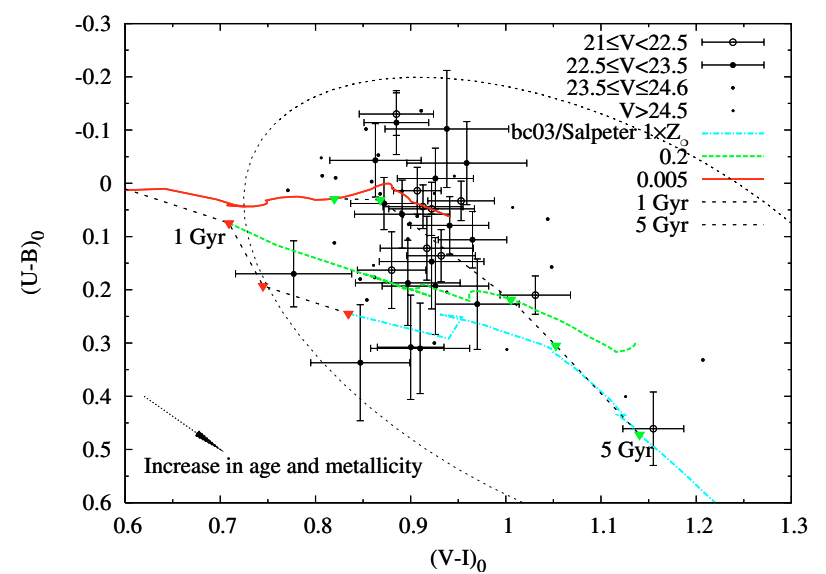

Fig. 11. The final selected globular cluster candidate sample. Dashed lines connect the SSP models with different metallicities (in solar units) for ages $1 \mathrm{Gyr}$ and $5 \mathrm{Gyr}$. With an ellipse is shown the GC color selection locus.

objects rejected by ACS (Sect. 3.3.1) the final number of GCCs is $N_{\mathrm{GC}}=60-4-18=38 \pm 8$, where the error encompasses the minimum and maximum number of contaminants quoted above.

\section{Analysis of the GC candidates' properties}

\subsection{Colors}

In a previous study of NGC $1427 \mathrm{~A}$ with the $2.5 \mathrm{~m}$ DuPont telescope at Las Campanas Observatory by Hilker et al. (1997) a selection of globular cluster candidates on the basis of their $V-I$ colors only was made. The introduction of the $U-B$ color in our study significantly improved the discrimination between globular clusters and highly reddened young star clusters. Only 7 globulars are in common between the current and the previous study. The larger number of GC candidates we derive is due to the significantly larger field of view of the FORS1 CCD detector and the much deeper exposures.

The final colors of the GC candidates are shown in Fig. 11. They cluster around low metallicities $(Z=0.0001$ and 0.0004$)$ and old or intermediate ages ( $\sim 5 \mathrm{Gyr})$ according to the Bruzual \& Charlot (2003) SSP models. With dashed lines the 1 and $5 \mathrm{Gyr}$ isochrones connecting SSP models with different metallicities are indicated. The photometric errors at these magnitudes and the convergence of the theoretical models prohibit accurate age and metallicity derivations for the individual globular cluster candidates. However, we can conclude that the ages and metallicities of the GC candidates around NGC 1427A as whole are comparable to the ages and metallicities of the old metal-poor Milky Way GCs.

The mean color $(V-I)_{0}=0.92 \pm 0.08$ of all GC candidates is similar to what was found for the globular cluster systems in dIrr, dSph and dE galaxies (Sharina et al. 2005; Seth et al. 2004; Lotz et al. 2004). This color corresponds to the blue GC population found in giant early-type galaxies and also obeys the relation between the host galaxy luminosity and the mean GC color (NGC 1427A: $M_{V}=-18.13$, see Sect. 4.3) (Harris et al. 2005; Peng et al. 2006; Larsen et al. 2001; Lotz et al. 2004).

In Fig. 12 we show the properties of the GC candidates in the $(U-B)_{0}$ vs. $(B-J)_{0}$ color-color plane. This color-color selection provides the best age/metallicity discrimination governed by the filters' transmission curves. The different line types in Fig. 12 represent Bruzual \& Charlot (2003) SSP models formetallicities 


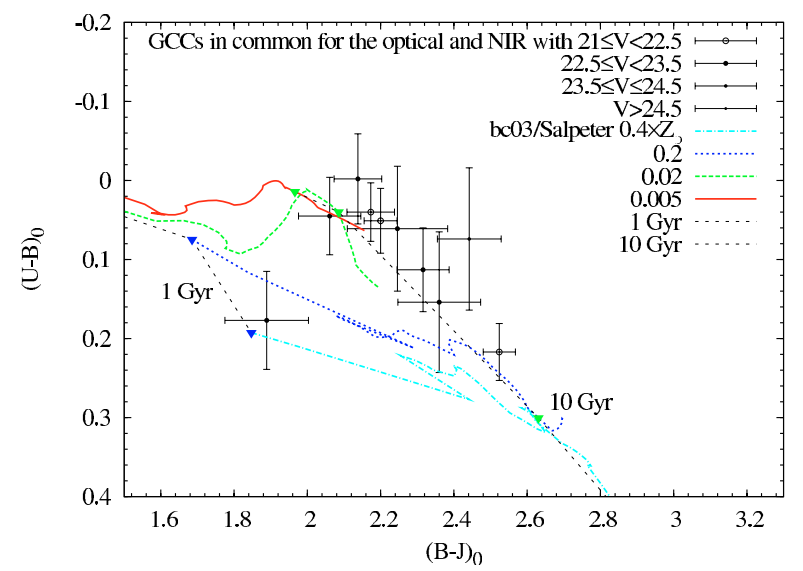

Fig. 12. Near IR color-color diagram for GC candidates in common for the FORS1 and ISAAC fields. Overplotted are the Bruzual \& Charlot (2003) SSP models for ages $\geq 1 \mathrm{Gyr}$ and metallicities lower than $0.4 \times Z_{\odot}$. With dotted lines the 1 and $10 \mathrm{Gyr}$ isochrones are indicated.

lower than $0.4 \times Z_{\odot}(Z=0.008)$. Although the IR images were not deep enough, a simple comparison with the 1 and $10 \mathrm{Gyr}$ isochrones (dotted lines in Fig. 12) reveals the predominantly old ages $(\geq 10 \mathrm{Gyr})$ and low metallicities $\left(\leq 0.2 \times Z_{\odot}\right)$ of the GC candidates. Hilker et al. (1997) showed that in integral properties NGC 1427A is similar to the Large Magellanic Cloud (LMC). Hence, taking into account the LMC metallicity of $Z=0.04 \times Z_{\odot}$ we can set the expected upper metallicity limit to this value. This is consistent with what is observed in Fig. 12.

\subsection{Luminosity function}

Figure 13 shows the NGC 1427A globular cluster luminosity function (GCLF) of the original and contamination corrected GCCs sample in bins of 0.4 mag. The dashed (green) histogram shows the GCLF for the original GC candidate sample while the solid (red) histogram represents the number counts corrected for contamination using the luminosity distribution of the contaminating objects in the outermost bins as estimated in Sect. 3.3.2.

Globular cluster luminosity functions have been studied for many galaxies (e.g. Ashman \& Zepf 1998; Kissler-Patig 2000; Richtler 2003; McLaughlin 2003, and ref's therein). It seems that the shape of the GCLF is universal among "old" GC systems and can be represented by a Gaussian or a $t_{5}$ function. In Fig. 13 the Gaussian fit to the corrected GCLF is shown. Due to the low number statistics we kept the width of the Gaussian fixed to $\sigma=1.2$ which is a typical value that fits most of the studied galaxies' GCLFs (e.g. Barmby et al. 2001). This choice of the dispersion gave $V_{\text {TO }}=23.61 \pm 0.10$ for the turnover magnitude of the BG/FG corrected sample. If we do not fix $\sigma$ we obtain $\sigma=1.45 \pm 0.16$ and $V_{\mathrm{TO}}=23.59 \pm 0.11$. The probability density function of the non-parametric density estimate using an Epanechnikov kernel (dotted line in Fig. 13) of the uncorrected GCLF has its maximum value at $V_{\text {TO }}=23.53 \pm 0.07 \mathrm{mag}$. The quoted uncertainties above include only the photometric errors and the uncertainty in the fit. The effect of the uncertainty in the number counts is reflected in the small difference in the final turnover magnitudes of the two distributions, which is within the errorbars.

Due to its universality the GCLF was frequently used as a distance indicator (for details see Richtler 2003), originally proposed by Hanes (1977). Assuming a universal turnover magnitude of $M_{V}=-7.40 \pm 0.11$, as derived for the MW GCS

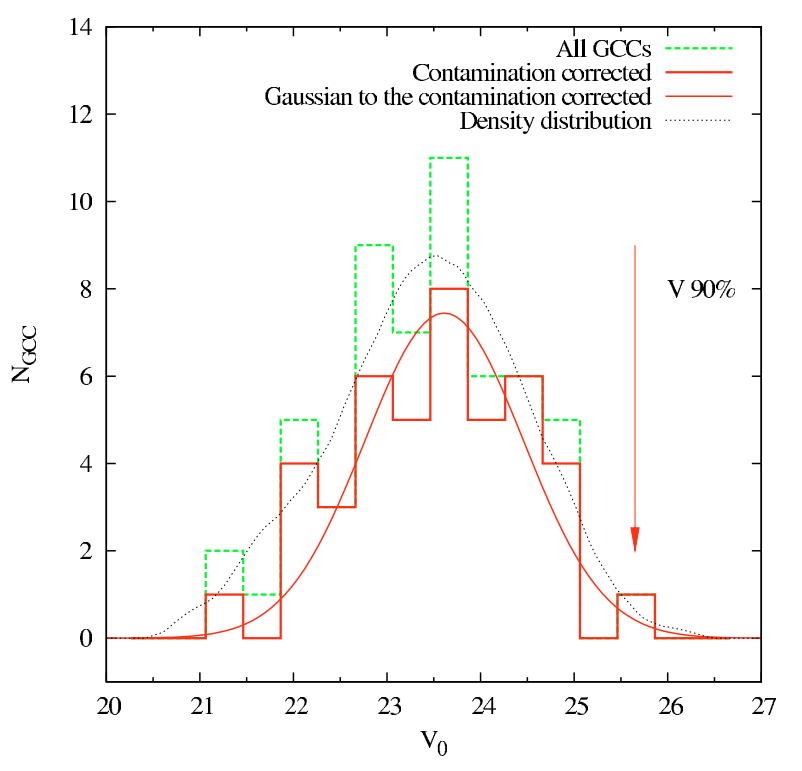

Fig. 13. NGC 1427A globular cluster luminosity function (GCLF). The solid (red) histogram represents the contamination corrected GCLF, the dashed histogram the uncorrected one. The curves are a Gaussian fit to the corrected GCLF (solid line) and the probability density estimate of the uncorrected GCLF using an Epanechnikov kernel (dotted curve). The arrow indicates the $90 \%$ completeness limit in $V$-band.

(Harris 2001) and those of other galaxies (e.g. Larsen et al. 2001; Ashman \& Zepf 1998, and ref's therein), the distance modulus to NGC 1427A is $(m-M)=31.01 \pm 0.21 \mathrm{mag}(15.9 \pm 1.6 \mathrm{Mpc})$. Our most shallow observation is in $U$-band with the $90 \%$ completeness limit at $U=26.21$, which is however still $\sim 1.4$ mag deeper than the expected $U_{\mathrm{TO}} \sim 24.8 \mathrm{mag}$ (assuming a mean $U-B \sim 0.1,(B-V) \simeq 0.7, M_{\mathrm{V}_{\text {Tо }}}=-7.40$, and a distance modulus of $31.39 \mathrm{mag})$. The last bin of the histogram $(V \simeq 25.8 \mathrm{mag})$ corresponds to $U \simeq 26.6$ mag which is approximately at the $85 \%$ completeness level. This implies a correction which makes the $V_{\text {TO }}$ fainter by $0.01 \mathrm{mag}$, which is well within the error and therefore we do not consider it further.

A recent study by Di Criscienzo et al. (2005) presents a new calibration of the GCLF performed on the basis of RR Lyrae in the MW, M31 and close-by galaxies as "primary" standard candles. They derive a GCLF turnover magnitudes of $V_{\mathrm{TO}}=-7.66 \pm 0.11$ for the MW, $V_{\mathrm{TO}}=-7.65 \pm 0.19$ for M31 and $V_{\mathrm{TO}}=-7.67 \pm 0.23$ for the other nearby galaxies. The weighted mean for the combined turnover magnitude then is $M_{\mathrm{V}_{\mathrm{T}}}=-7.66 \pm 0.09 \mathrm{mag}$. If we apply this value to the GCS of NGC 1427A we obtain a distance modulus of $(m-M)=31.25 \pm 0.20 \mathrm{mag}(17.8 \pm 1.7 \mathrm{Mpc})$.

Finally, independently of which absolute GCLF turnover magnitude is adopted, the direct comparison of the apparent GCLF turnovers for NGC $1399\left(V_{\mathrm{TO}}=24.01 \pm 0.1\right.$, Dirsch et al. 2003) and NGC 1427A places the latter approximately at $3.2 \pm 2.5$ (statistic) \pm 1.6 (systematic) Mpc in front of NGC 1399 . The statistical error is determined from the photometric and our fitting routine uncertainities of the turnover magnitude; the systematic error includes the uncertainties of the absolute magnitude and the GCLF method itself (Richtler 2003). It should be noted that the giant elliptical NGC 1404 is located even closer in projection to NGC 1427A. Unfortunately, the GCLF turnover magnitude for NGC 1404 (Blakeslee \& Tonry 1996; Richtler et al. 1992) is not as accurately determined as that of NGC 1399, and thus we can not perform a similar comparison. 


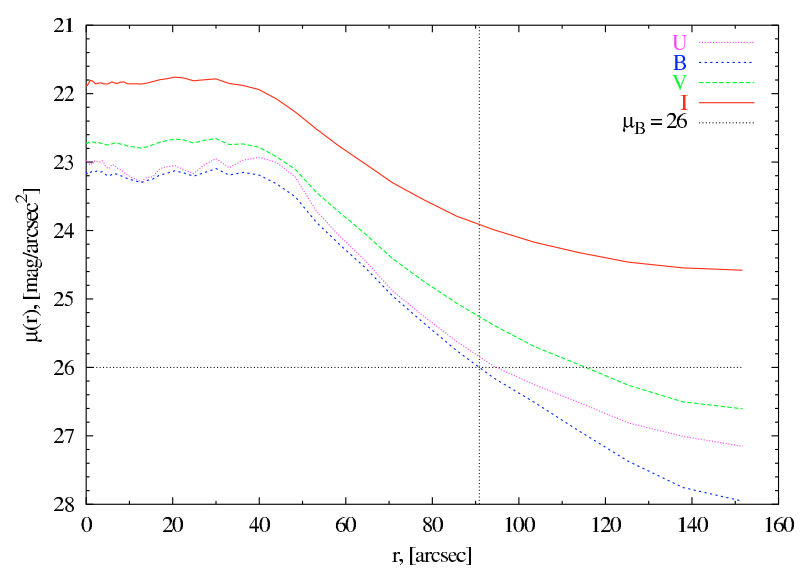

Fig. 14. The NGC 1427A surface brightness profile in $U, B, V$, and $I$-bands. The vertical dotted line indicates the radius of the $\mu_{B}=$ $26 \mathrm{mag} / \operatorname{arcsec}^{2}$ isophote, at which the $V$-band $\mu_{V}=25.6 \mathrm{mag} \operatorname{arcsec}^{-2}$ was determined and plotted in Fig. 9.

\subsection{Specific frequency}

The "specific frequency" $\left(S_{\mathrm{N}}\right)$ is a quantity introduced by Harris $\&$ van den Bergh (1981) to intercompare the GCSs between different elliptical galaxies. The $S_{\mathrm{N}}$ is defined as the total GC $\left(N_{\mathrm{GC}}\right)$ population normalized to a galaxy luminosity of $M_{V}=$ -15 mag:

$S_{\mathrm{N}}=N_{\mathrm{GC}} 10^{0.4\left(M_{V}+15\right)}$.

In order to derive the specific frequency properly one needs to correct for background and foreground contamination, the unobserved part of the GCS (and the parent galaxy) and the completeness of the observations at the faint end of the GCLF. One of the most important issues is that $S_{\mathrm{N}}$ should be derived from GC counts and total galaxy light both estimated within the same area. Otherwise systematics in the GCS and/or galaxy profile could affect the estimated $S_{\mathrm{N}}$ values (Dirsch et al. 2003; Ostrov et al. 1998).

Having the $V$-band $90 \%$ completeness limit $\sim 1.5 \mathrm{mag}$ (see Sect. 4.2 and Fig. 3) beyond the expected GCLF turnover magnitude, completeness corrections are insignificant for the estimate of $S_{\mathrm{N}}$. Due to the complete coverage of the galaxy including its outer parts we do not need to make any geometrical corrections. In order to measure the total galaxy light of NGC 1427A we used the IRAF/STSDAS task ELLIPSE on the background subtracted images. The bright, obvious background and foreground sources were masked out. Due to the complex morphology of this irregular galaxy the definition of its center was a complicated task. We adopted the center which best represents the diffuse galaxy light distribution at the radius of the $\mu_{B}=26 \mathrm{mag} \operatorname{arcsec}^{-2}$ isophote (marked with " $x$ " in Fig. 9). This is the common center for the both circles drawn in Fig. 9 in which we estimate the $S_{\mathrm{N}}$. The $V$-band isophote $\left(\mu_{V}=25.6 \mathrm{mag} \operatorname{arcsec}^{-2}\right)$ shown in Fig. 9 is determined at the radius of the $B$-band $\mu_{B}=26$ isophote (compare with Fig. 14). Another approach was to fit the center on the 41 pix ring aperture smoothed image (see Sect. 2.2) using the IRAF centroid algorithm. The result of this centering is shown with a "+" symbol in Fig. 9. Based on the NGC 1427A ionized gas kinematics, Chanamé et al. (2000) defined a kinematical center which is indicated with a diamond symbol in Fig. 9. The latter two center coordinates are offset with respect to the center defined by the diffuse galaxy light while the adopted geometrical $V$-band isophote center does not only represent the center of the diffuse galaxy light (stars) but also is the most symmetrical center of the GC candidate distribution around NGC 1427A. In order to check the centering effect we measured the differences of the total galaxy magnitudes assuming the different centers and found negligible deviations below $2 \%$ (0.02 mag). Thus we adopted the isophotal center as a good enough approximation. In Fig. 14 the galaxy surface brightness profiles for $U, B, V$, and $I$ are shown. With the dotted lines the extension of the $\mu_{B}=26$ isophote (at $r_{1}=900^{\prime} 9$ ) is indicated, for which the total magnitude $V_{\text {TOT,1 }}=13.06 \pm 0.02$ mag was determined. As can be seen, the surface brightness profile reaches the background level in the $I$-band approximately at $r_{2}=151^{\prime \prime}$. 7 . We adopt this truncation radius for the other pass-bands. The total magnitude at that radius would be $V_{\mathrm{TOT}, 2}=12.88 \pm 0.02 \mathrm{mag}$. The determination of $S_{\mathrm{N}}$ for both radii will give us information on the radial dependence of the specific frequency in NGC 1427A. Inside $r_{1}$ and $r_{2}$ we count 23 and 40, respectively.

The correction for contamination is based on the determined level of $0.61 \mathrm{objects} / \mathrm{arcsec}^{-2}$ (see Sect. 3.3.2). Thus, for the two radii we determine a correction of 4 and 12 contaminants leading to $N_{\mathrm{GC}}=19$ and 28 , respectively.

Using the adopted distance to Fornax we obtain $M_{V, 1}=$ $-18.33 \pm 0.20$ and $M_{V, 2}=-18.51 \pm 0.20 \mathrm{mag}$. The calculated specific frequencies are $S_{\mathrm{N}}=0.88$ and $1.09 \pm 0.23$. Using the distance modulus determined from the GCLF turnover we compute $M_{V, 1}=-17.95 \pm 0.21$ and $M_{V, 2}=-18.13 \pm 0.21 \mathrm{mag}$, which results in the following specific frequencies, $S_{\mathrm{N}}=1.3$ and $1.6 \pm 0.23$ for the smaller and larger aperture, respectively. These $S_{\mathrm{N}}$-values should be considered as lower limits for NGC 1427A's specific frequency since an age-fading of the galaxy light should be applied to compare it with the $S_{\mathrm{N}}$ of giant early-type galaxies. As Miller et al. (1998) estimated, the final total $V$ magnitude of a galaxy that is forming stars for $5 \mathrm{Gyr}$ at constant rate and then is fading for another $5 \mathrm{Gyr}$ will be fainter by $\sim 1.5 \mathrm{mag}$ as during the star forming phase. Assuming that the number of old GCs is conserved, this luminosity evolution will increase the $S_{\mathrm{N}}$ by a factor of $\sim 4$ during this time. Also, the number of the old GCs could increase of the most massive and/or compact young star clusters, present in the starburst regions, survive the disruptive dynamical evolution.

The $S_{\mathrm{N}}$ value measured here $(\geq 1)$ is higher than typical values for dIrrs (e.g. $S_{\mathrm{N}} \simeq 0.5$ for LMC, Harris 1991) in the Local Group. However, our estimates are comparable with results by Seth et al. (2004) for dIrrs in the Virgo and Fornax clusters, $S_{\mathrm{N}}>2$, which suggests that the environment influences the GC formation efficiency in dIrr galaxies.

Another approach avoiding the uncertain galaxy light agefading estimate is to use the $T$ parameter proposed by Zepf \& Ashman (1993) which relates the total GC number to the total galaxy mass:

$$
T=\frac{N_{\mathrm{GC}}}{M_{\mathrm{G}} / 10^{9} M_{\odot}}
$$

where $M_{\mathrm{G}}$ is the total galaxy mass. Chanamé et al. (2000), using a rigid-body rotation model, determined the NGC 1427A angular velocity of $\omega=12.8 \pm 1 \mathrm{~km} \mathrm{~s}^{-1} \mathrm{kpc}^{-1}$. They derive a lower limit of the dynamical mass of $M_{\mathrm{G}}=(9 \pm 3) \times 10^{9} M_{\odot}$ within $6.2 \mathrm{kpc}$ radius corresponding to $\mu_{V}=24.7 \mathrm{mag} / \mathrm{arcsec}^{2}$. This isophote corresponds to 77!' 8 distance from the dynamical center. Within this aperture, after correction for the contamination of 3 objects, we count $19 \mathrm{GC}$ candidates and thus obtain the local $T$ value of $2.1 \pm 1$. However, the total $\mathrm{T}$ value should be used for comparison. We calculate at $r_{2}=151: 7 \simeq 12 \mathrm{kpc}$ the NGC 1427A dynamical mass of $M_{\mathrm{G}}=(6 \pm 3) \times 10^{10} M_{\odot}$ and 
$N_{\mathrm{GC}}=28$, which gives $T=0.5 \pm 1$. This GC formation efficiency per unit mass is smaller but comparable to the $T$ values of spiral galaxies (e.g., Chandar et al. 2004; Goudfrooij et al. 2003; Barmby \& Huchra 2001; Kissler-Patig et al. 1999).

\section{Conclusions}

Based on deep VLT observations, we study the old GC population of the dwarf irregular galaxy NGC 1427A. The results of our analysis are as follows. After applying various selection criteria, namely color cuts, point source selection, exclusion of $\mathrm{H} \alpha$-emitting sources, and a visual inspection of HST/ACS images resulted in 56 GCCs. Accounting for contamination (Sect. 3.3) this finally leads to $38 \pm 8$ globular cluster candidates, likely associated with NGC 1427A. Their radial number density distribution is concentrated towards the galaxy center, which suggests that they belong to this galaxy. The colors of the selected GC candidates show that most of them are metalpoor $\left(Z \leq 0.08 \times Z_{\odot}\right)$, old globular clusters $(\geq 5 \mathrm{Gyr})$ according to comparisons with simple stellar population models (Bruzual \& Charlot 2003) and colors of the old GCs in the Milky Way. This is in agreement with results from previous investigations on GCS in dIrr, dSph and dE galaxies (e.g. Sharina et al. 2005; Seth et al. 2004; Lotz et al. 2004), which show that the GCSs in these systems share similar properties, hence similar early star formation histories.

Using the GCLF turnover magnitude as a standard candle, we estimated the distance to NGC 1427A for the first time. We derive a distance modulus of $(m-M)=31.01 \pm 0.21 \mathrm{mag}$ $(15.9 \pm 1.6 \mathrm{Mpc})$ taking the measured GCLF turnover of $V_{\mathrm{TO}}=$ $23.61 \pm 0.11$ and assuming $M_{\mathrm{V}_{\text {Tо }}}=-7.40 \pm 0.11$ as universal (Harris 2001). If we use the RR Lyrae calibrated GCLF $M_{\mathrm{V}_{\text {Tо }}}=$ $-7.66 \pm 0.09$ (Di Criscienzo et al. 2005) we obtain $(m-M)=$ $31.25 \pm 0.20 \mathrm{mag}(17.8 \pm 1.5 \mathrm{Mpc})$. The analysis shows that NGC $1427 \mathrm{~A}$ is placed $3.2 \pm 2.5$ (statistic) \pm 1.6 (systematic) Mpc in front of the giant cD galaxy NGC 1399. If NGC 1427A really is located more than $2-3 \mathrm{Mpc}$ away from the cluster center, the suggested interaction of NGC 1427A with the dense intracluster medium triggering the intense star formation activity would then probably occur at large cluster-centric radii. This result, however, should be taken with precaution considering the uncertainties involved in the GCLF turnover point and the possible systematics in the method itself of about $0.2 \mathrm{mag}$ (for a review see Richtler 2003). More importantly, a precise analysis of the relationship between NGC 1427A and the cluster environment must involve the giant elliptical NGC 1404 as well. We are currently prevented to do this due to the large uncertainties in the relevant distances. However, our relative distance result supports the Drinkwater et al. (2001) finding that the kinematically distinct population of infalling dwarf galaxies in Fornax shows an extended spatial distribution.

We obtained a present-day specific frequency of $S_{\mathrm{N}}=1.6 \pm$ 0.23 . However, this galaxy still is actively forming stars. Hence, an age fading of the galaxy's light should be applied prior to compare its $S_{\mathrm{N}}$ value to those of "old" early-type galaxies. Applying such a correction (according to Miller et al. 1998) would lead to a $S_{\mathrm{N}}$ value of $\sim 7$ after passive evolution for a few Gyr. The large $S_{\mathrm{N}}$ value suggests that dIrrs could contribute to the blue GC population and its total numbers in giant early-type galaxies through dissipationless merging or accretion. Since they are still actively forming stars and star clusters, they may also contribute to the red GC population by some newlyformed GCs. Those clusters might form out of material that was enriched during the starburst event, probably triggered by the interaction with the cluster environment.

Given the current paucity of deep imaging studies of dIrr galaxies combined with the fact that they are likely building blocks of giant galaxies in the context of the hierarchical merging scenario, we suggest that detailed studies of dIrr galaxies in a range of environments be undertaken to establish their range of GCS properties as a function of environment density. This should provide important constraints on the formation of these galaxies as well as on their global impact on galaxy formation and evolution.

Acknowledgements. I.G. acknowledges the partial support from the DFG Graduate Research School 787 on "Galaxy Groups as Laboratories for Baryonic and Dark Matter". I.G. and P.G. would like to thank the director of STScI for the award of a graduate studentship funded by the Director's Discretionary Research Fund. The authors also gratefully acknowledge the valuable comments and discussions with Rupali Chandar, Michael Drinkwater and Klaas de Boer. JC wishes to express his gratitude to the faculty at the Department of Astronomy of The Ohio State University for their continuous support to this external collaboration. AR and LF would like to acknowledge the support from FONDECYT Regular Grant 1020840 and the FONDAP center for Astrophysics. Finally, we wish to thank the referee for making a number of helpful comments that helped to improve the present paper.

\section{References}

Anders, P., Bissantz, N., Fritze-v. Alvensleben, U., \& de Grijs, R. 2004, MNRAS, 347, 196 Ashman, K. M., \& Zepf, S. E. 1992, ApJ, 384, 50

Ashman, K. M., \& Zepf, S. E. 1998, Globular cluster systems (Cambridge University Press) Barmby, P., \& Huchra, J. P. 2001, AJ, 122, 2458

Barmby, P., Huchra, J. P., \& Brodie, J. P. 2001, AJ, 121, 1482

Bassino, L. P., Cellone, S. A., Forte, J. C., \& Dirsch, B. 2003, A\&A, 399, 489

Beasley, M. A., Baugh, C. M., Forbes, D. A., Sharples, R. M., \& Frenk, C. S. 2002, MNRAS, 333,383

Blakeslee, J. P., \& Tonry, J. L. 1996, ApJ, 465, L19

Böker, T., Calzetti, D., Sparks, W., et al. 1999, ApJS, 124, 95

Bruzual, G., \& Charlot, S. 2003, MNRAS, 344, 1000

Bureau, M., Mould, J. R., \& Staveley-Smith, L. 1996, ApJ, 463, 60

Cardelli, J. A., Clayton, G. C., \& Mathis, J. S. 1989, ApJ, 345, 245

Carney, B. W., \& Harris, W. E. 1998, ed. L. Labhardt, \& B. Binggeli, Star Clusters (Berlin: Springer-Verlag), 2001, p. 8, 2000 update

Cellone, S. A., \& Forte, J. C. 1997, AJ, 113, 1239

Chanamé, J., Infante, L., \& Reisenegger, A. 2000, ApJ, 530, 96

Chandar, R., Whitmore, B., \& Lee, M. G. 2004, ApJ, 611, 220

Côté, P., Marzke, R. O., \& West, M. J. 1998, ApJ, 501, 554

Côté, P., West, M. J., \& Marzke, R. O. 2002, ApJ, 567, 853

Di Criscienzo, M., Caputo, F., Marconi, M., \& Musella, I. 2005, ArXiv Astrophysics e-prints Dirsch, B., Richtler, T., Geisler, D., et al. 2003, A\&A, 125, 1908

Drinkwater, M. J., Gregg, M. D., \& Colless, M. 2001, ApJ, 548, L139

Elmegreen, B. G. 1999, Ap\&SS, 269, 469

Ferguson, H. C., \& Sandage, A. 1990, AJ, 100, 1

Forbes, D. A., Brodie, J. P., \& Grillmair, C. J. 1997, AJ, 113, 1652

Forbes, D. A., Grillmair, C. J., Williger, G. M., Elson, R. A. W., \& Brodie, J. P. 1998, MNRAS, 293, 325

Freedman, W. L., Madore, B. F., Gibson, B. K., et al. 2001, ApJ, 553, 47 Goudfrooij, P., Strader, J., Brenneman, L., et al. 2003, MNRAS, 343, 665 Hamuy, M., Walker, A. R., Suntzeff, N. B., et al. 1992, PASP, 104, 533

Hamuy, M., Suntzeff, N. B., Heathcote, S. R., et al. 1994, PASP, 106, 566

Hanes, D. A. 1977, MNRAS, 180, 309

Harris, W. E. 1991, ARA\&A, 29, 543

Harris, W. E. 1996, AJ, 12, 1487

Harris, W. E. 2001, Globular cluster systems, in Saas-Fee Advanced Course 28: Star Clusters, 223

Harris, W. E. 2003, Issues in E Galaxy Formation: Many Problems, Few Solutions, in Extragalactic Globular Cluster Systems, 317

Harris, W. E., \& van den Bergh, S. 1981, AJ, 86, 1627

Harris, W. E., Whitmore, B. C., Karakla, D., et al. 2005, ApJ, 636, 90

Heidt, J., Appenzeller, I., Gabasch, A., et al. 2003, A\&A, 398, 49

Hilker, M., Bomans, D. J., Infante, L., \& Kissler-Patig, M. 1997, A\&A, 327, 562

Hilker, M., Infante, L., \& Richtler, T. 1999, A\&AS, 138, 55

Hunter, D. A., \& Elmegreen, B. G. 2004, AJ, 128, 2170

James, P. A., Shane, N. S., Knapen, J. H., Etherton, J., \& Percival, S. M. 2005, A\&A, 429, 851

Jordán, A., Côté, P., Blakeslee, J. P., et al. 2005, ApJ, 634, 1002

Kissler-Patig, M. 2000, Rev. Mod. Astron., 13, 13

Kissler-Patig, M., Ashman, K. M., Zepf, S. E., \& Freeman, K. C. 1999, AJ, 118, 197

Knapen, J. H., Stedman, S., Bramich, D. M., Folkes, S. L., \& Bradley, T. R. 2004, A\&A, 426,1135 
Koekemoer, A. M., Fruchter, A. S., Hook, R. N., \& Hack, W. 2002, MultiDrizzle: An Integrated Pyraf Script for Registering, Cleaning and Combining Images, in The 2002 HST Calibration Workshop: Hubble after the Installation of the ACS and the NICMOS Cooling System, Proceedings of a Workshop held at the Space Telescope Science Institute, Baltimore, Maryland, October 17 and 18, 2002, ed. S. Arribas, A. Koekemoer, \& B. Whitmore, Baltimore, MD: Space Telescope Science Institute, 339

Kundu, A., \& Whitmore, B. C. 2001, AJ, 121, 2950

Landolt, A. U. 1992, AJ, 104, 340

Larsen, S. S., Brodie, J. P., Huchra, J. P., Forbes, D. A., \& Grillmair, C. J. 2001, AJ, 121, 2974

Lotz, J. M., Miller, B. W., \& Ferguson, H. C. 2004, ApJ, 613, 262

Mackey, A. D., \& van den Bergh, S. 2005, MNRAS, 360, 631

McLaughlin, D. E. 2003, The Globular Cluster Luminosity Function, in Extragalactic Globular Cluster Systems, 329

Miller, B. W., Lotz, J. M., Ferguson, H. C., Stiavelli, M., \& Whitmore, B. C. 1998, ApJ, $508, \mathrm{~L} 133$

Ostrov, P. G., Forte, J. C., \& Geisler, D. 1998, AJ, 116, 2854

Peng, E. W., Jordan, A., Cote, P., et al. 2006, ApJ, 639, 95
Puzia, T. H., Kissler-Patig, M., Thomas, D., et al. 2004, A\&A, 415, 123

Richtler, T. 2003, Stellar Candles for the Extragalactic Distance Scale, LNP, 635, 281

Richtler, T., Grebel, E. K., Domgoergen, H., Hilker, M., \& Kissler, M. 1992, A\&A, 264, 25

Robin, A. C., Reylé, C., Derriére, S., \& Picaud, S. 2003, A\&A, 409, 523

Sandage, A. 2005, ARA\&A, 43, 581

Schlegel, D. J., Finkbeiner, D. P., \& Davis, M. 1998, ApJ, 500, 525

Schweizer, F. 1987, Star formation in colliding and merging galaxies, in Nearly Normal Galaxies, From the Planck Time to the Present, 18

Seth, A., Olsen, K., Miller, B., Lotz, J., \& Telford, R. 2004, AJ, 127, 798

Sharina, M. E., Puzia, T. H., \& Makarov, D. I. 2005, A\&A, 442, 85

Stetson, P. B. 1987, PASP, 99, 191

van den Bergh, S. 1993, ApJ, 411, 178

van den Bergh, S. 2000, PASP, 112, 932

van den Bergh, S., \& Mackey, A. D. 2004, MNRAS, 354, 713

van Dokkum, P. G. 2001, PASP, 113, 1420

Yahagi, H., \& Bekki, K. 2005, MNRAS, 364, L86

Zepf, S. E., \& Ashman, K. M. 1993, MNRAS, 264, 61 